\title{
REBA
}

Revista Boliviana de Administración

Volumen 3 | Número 1 Enero - junio 2021 | ISSN: 2710 - 0863 / ISNN-L: 2710 - 0863| Pág. 13 - 21/ https://revistareba.org/

https://doi.org/10.33996/reba.v3i1.2

\section{Gestión de riesgos en el ambiente laboral de las instituciones públicas de educación universitaria del municipio Cabimas}

\section{Risk management in work environment of the public institutions of university education of Cabimas municipality}

\author{
४ Robert Rodríguez \\ ORCID: 0000-0001-9366-1702
}

rrobert13@gmail.com

Universidad del Zulia

Maracaibo - Venezuela

Artículo recibido en octubre 2020 / Arbitrado en noviembre 2020 / Aceptado en noviembre 2020 / Publicado en enero 2021

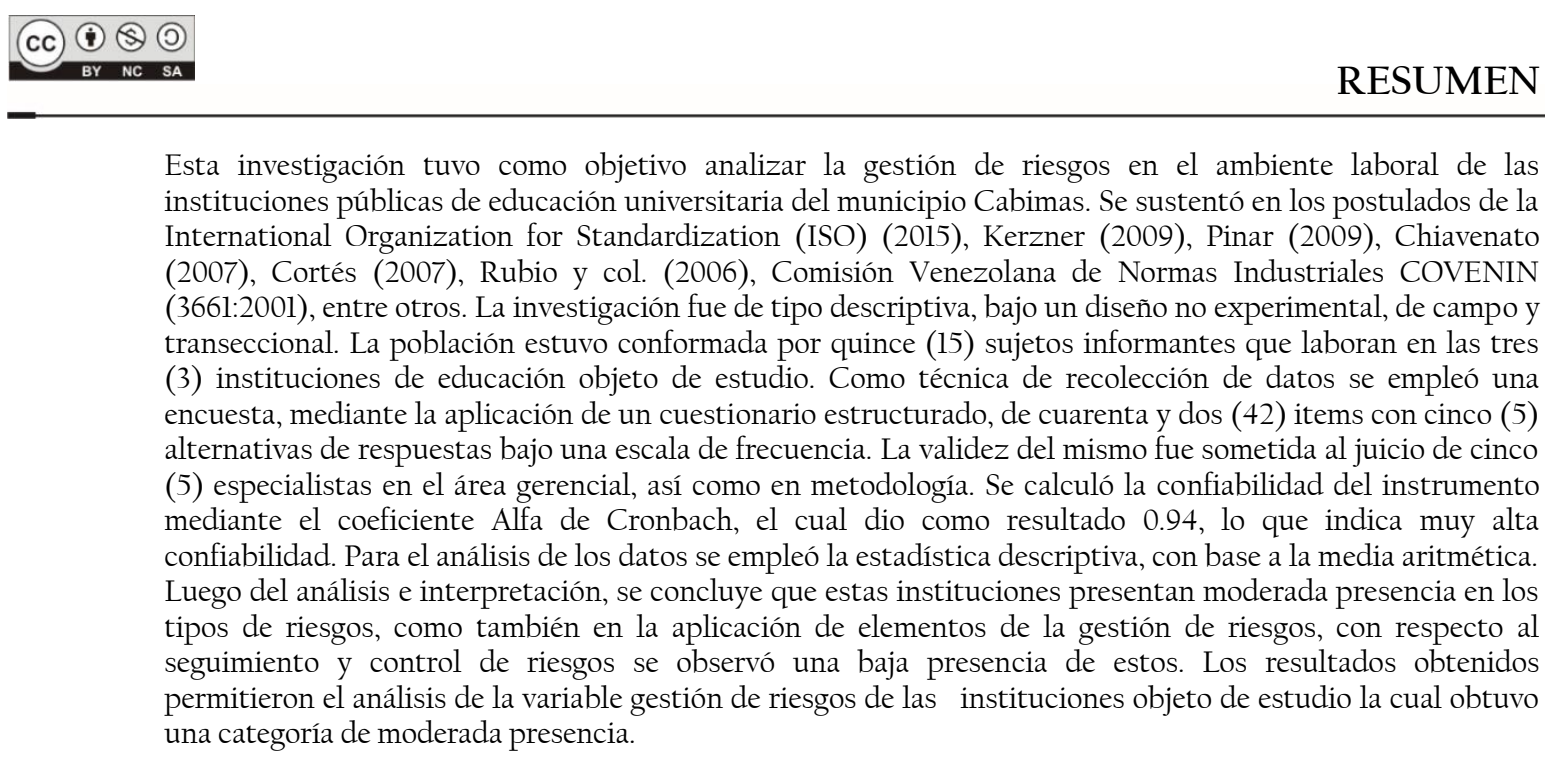

Palabras clave: Gestión de riesgos, instituciones públicas de educación, riesgos, elementos de prevención, seguimiento y control

\section{ABSTRACT}

This research aims was to analyze the risk management in work environment of the public institutions of university education of Cabimas municipality. This was based on the postulates of International Organization for Standardization (ISO) (2015), Kerzner (2009), Pinar (2009), Chiavenato (2007), Cortés (2007), Rubio y col. (2006), Venezuelan Industrial Standards Commission COVENIN (3661:2001), among others. The research was descriptive, under a non-experimental field design, and transectional. The population was conformed by fifteen (15) Informants subjects that work in the three (3) educational institutions object of study. As data collection technique a survey was used, through the application of a structured questionnaire, of forty-two (42) items with five (5) response alternatives under a frequency scale. The validity of the same was submitted to the trial of five (5) specialists in the management area, as well as methodology. The reliability of instrument was estimated using the Alpha Cronbach coefficient, obtained a coefficient of 0.94 , it showed a very high reliability. For the analysis of the data was used the descriptive statistics. After analysis and interpretation, it is concluded that these institutions have a moderate presence in the types of risks, as well as in the application of elements of risk management, with respect to the monitoring and control of risks was observed a low presence of these. The results of this research allowed the analysis of the risk management in these public institutions which obtained a category of moderate presence.

Keywords: risk management, public institutions of education, risk, elements of prevention, monitoring and control 


\section{INTRODUCCIÓN}

Las organizaciones, no importa cuál sea su actividad y tamaño, afrontan una serie de riesgos que pueden afectar su normal desenvolvimiento. Todas las actividades de una organización están sometidas de forma permanente a una serie de amenazas, lo cual las hace vulnerables, comprometiendo su estabilidad. Accidentes operacionales, enfermedades, incendios, catástrofes naturales, entre otros, son una muestra de este panorama. La gestión de riesgos ya no se limita al enfoque financiero tradicional, ahora ésta contempla aspectos muy variados como la seguridad, y estrategias para prevenir, reducir o transferir riesgos.

Aunque la gestión de riesgos es un concepto intuitivo en el contexto de las empresas, la estandarización de los procesos, ha perfilado a la gestión de riesgos como una estrategia a ser aplicada en todo tipo de organización. Las instituciones públicas de educación universitaria pueden ser consideradas como organizaciones que brindan conocimiento a los estudiantes y un área laboral para los empleados de la institución. Por lo tanto, uno de los retos que deben afrontar las instituciones públicas de educación universitaria es implementar un sistema de gestión de riesgos que facilite la administración de su actividad y que al mismo tiempo fortalezca sus capacidades para prevenir sus vulnerabilidades.

Es así, como en la actualidad las organizaciones buscan implantar sistemas de gestión de riesgos que les permitan no solo controlar los riesgos asociados a las actividades, sino también, cumplir con las leyes, reglamentos y normas en materia de prevención de riesgos, para asegurar la conformidad con todo lo establecido en el marco legal aplicable a cualquier actividad productiva o de servicio, cualquiera sea su naturaleza, pública o privada del país.

A nivel mundial el papel que juegan las instituciones públicas de educación universitaria en la sociedad varían según la región, la cultura o la institucionalidad de la misma, pero en general se puede dar dos finalidades de estas instituciones. La primera seria suministrar los conocimientos necesarios a los estudiantes para que obtengan un diploma, la segunda seria brindar un ambiente donde el estudiante aprenda a ser autónomo emocionalmente, a relacionarse con gente de diferentes orígenes, y a desarrollarse como persona; pero al final, todo esto se resume en, proporcionar las herramientas necesarias para el desarrollo intelectual y personal del capital humano.

En este sentido, la Ley de Educación Universitaria, reseñada en Gaceta Oficial $\mathrm{N}^{\circ}$ 1.429 (Extraordinaria) de fecha 8 de septiembre de 1970, define a las instituciones de educación universitaria de la siguiente manera.

Se entiende por recinto universitario
el espacio precisamente delimitado
y previamente destinado a la
realización de funciones docentes,
de investigación, académica, de
extensión o administrativa, propia
de la Institución. Corresponde a las
autoridades nacionales y locales la
vigilancia de las avenidas, calles y
otros sitios abiertos al libre acceso y
circulación, y la protección y
seguridad de los edificios y
construcciones situados dentro de
las áreas donde funcionen las
universidades, y las demás medidas
que fueren necesarias a los fines de
salvaguardar y garantizar el orden
público y la seguridad de las
personas y de los bienes, aun
cuando éstos formen parte del
patrimonio de la universidad.

Basados en la premisa de la seguridad de las personas y de los bienes, de la Ley de Educación Universitaria (1970), se establece la importancia que tiene la gestión de riesgos en el ambiente laboral de las instituciones públicas de educación universitaria, ya que les permite organizarse, reducir los riesgos y tomar medidas para poder prevenir y responder de manera adecuada a los 
desastres o incidentes que se presenten en la institución. Según Kerzner (2009), la gestión de riesgos es el acto o práctica de tratar con riesgos, esto incluye planificación para riesgos, evaluación de riesgos, desarrollo de opciones de manejo de riesgos, y monitoreo de riesgos para determinar cómo se presentan y evolucionan los mismos.

A tal efecto, el Programa de las Naciones Unidas para el Desarrollo, PNUD, (2012), plantea que aunque muchas personas están conscientes de las trágicas consecuencias que pueden provocar cualquier desastre o incidente en su entorno, son pocos los que se dan cuenta que se puede hacer algo para reducir sus efectos, de allí que a nivel mundial se han acentuado los problemas que causan cada día eventos no deseados que generan pérdidas económicas, lesiones y/ o pérdidas humanas.

Cabe destacar, que América Latina no es la excepción, encontrándose específicamente en Venezuela, que su ubicación geográfica es una zona susceptible a riesgos de desastres naturales como lluvias tropicales, inundaciones, terremotos, e incendios forestales, pero también es susceptible a otro tipo de riesgos, los antrópicos, que causan mayores pérdidas anuales; entre los que se pueden mencionar los riesgos de hurto, terrorismo, incendios, violencia, entre otros. En el presente se busca sensibilizar y formar a la población para establecer y fortalecer una cultura de prevención basada en la gestión de riesgo, en donde la protección individual y colectiva sea un valor indispensable para la vida que a su vez también sirva de herramienta para dar respuestas de planificación y desarrollo en todos los ámbitos.

Según Barbat (2005), para corregir las causas del riesgo mediante acciones de intervención de la vulnerabilidad y mediante el fortalecimiento de la capacidad de gestión del riesgo en todas sus modalidades, es necesario identificar el riesgo existente, como también las posibilidades de generación de nuevos riesgos. Esto implica dimensionar o medir el riesgo, monitorearlo con el fin de determinar la efectividad y eficiencia de las medidas de intervención. Además de la evaluación y el seguimiento por parte de los diversos actores sociales y órganos de decisión responsable de la gestión. Es decir, es necesario hacer manifiesto el riesgo, socializarlo e identificar su causa. En consecuencia, dicha evaluación debe realizarse utilizando herramientas apropiadas e idóneas que faciliten la comprensión del problema y orienten la toma de decisiones.

En el caso de las instituciones objeto de estudio, es importante destacar que, luego de realizar una serie de entrevistas a personas que laboran en el área de seguridad, además de evaluar las infraestructuras de éstas se pudo constatar que las condiciones de seguridad en las diferentes áreas de estudio, y de esparcimiento no son apropiadas, evidenciándose carencia de programas de gestión de riesgos, así como ausencia de planes de motivación y sistemas de control lo que genera déficit en la cultura de prevención de riesgos. También se debe resaltar la ocurrencia de varios accidentes tanto naturales como antrópicos que causaron pérdidas humanas y materiales, los cuales con la aplicación apropiada de una gestión de riesgos oportuna, habrían evitado.

Por otra parte, en estas entrevistas se detectó que en estas instituciones no se realizan evaluaciones de riesgos, o monitoreo de éstos para determinar cómo han cambiado los mismos, y tomar así las medidas necesarias para evitarlos o controlarlos. De allí que para las instituciones públicas de educación universitaria del municipio Cabimas sea de suma urgencia e importancia implementar una gestión de riesgo para dar así cumplimiento a la Ley Orgánica de Prevención, Condiciones y Medio Ambiente de Trabajo LOPCYMAT (2005), que en su artículo 53 estipula:

Los trabajadores y las trabajadoras tendrán derecho a desarrollar sus labores en un ambiente de trabajo adecuado y 
propicio para el pleno ejercicio de sus facultades físicas y mentales, y que garantice condiciones de seguridad, salud, y bienestar adecuadas.

Es así, como para dar a conocer la importancia de la gestión de riesgos, esta investigación permite su descripción en el ambiente laboral de las instituciones públicas de educación universitaria del municipio Cabimas y en virtud de los planteamientos antes expuestos se plantea la siguiente interrogante de investigación: ¿Cómo es la gestión de riesgos en el ambiente laboral de las instituciones públicas de educación universitaria del municipio Cabimas?

\section{MATERIALES Y METODOS}

El presente estudio se ubicó dentro del tipo de investigación descriptiva, enmarcada en un diseño no experimental, de campo y transeccional. La población objeto de estudio estuvo constituida por 15 sujetos informantes que laboran en las tres (3) instituciones públicas de educación universitaria objeto de estudio, quienes desempeñaban cargos de dirección, coordinadores y delegados de prevención en cada institución. Tomando en cuenta que el número de sujetos es finito, $\mathrm{y}$ accesible estadísticamente, no se realizó plan de muestreo, en este caso, se utilizó la técnica de censo poblacional.

Para la recolección de los datos se utilizó la técnica de la encuesta, y como instrumento se construyó un cuestionario, tipo escala de frecuencia de cinco (5) categorías. Para determinar la confiabilidad del instrumento se utilizó el método de coeficiente de Alfa Cronbach, obteniéndose un coeficiente de 0,94 que indica una muy alta confiabilidad. El análisis de los datos se realizó en forma cuantitativa, y para su análisis se utilizó la estadística descriptiva, trabajando con la media aritmética.

La tabla 1 presenta el baremo de interpretación, el cual se diseñó con el propósito de categorizar los resultados obtenidos del tratamiento estadístico luego de la aplicación del instrumento.

Tabla 1. Baremo de interpretación

\begin{tabular}{ccc}
\hline Intervalo & Categoría & Descripción \\
\hline $\mathbf{4 . 2 1}-\mathbf{5 . 0 0}$ & Muy alta & Indica una muy alta presencia de la variable, dimensión, indicador. \\
$\mathbf{3 . 4 1 - 4 . 2 0}$ & Alta & Indica una alta presencia de la variable, dimensión, indicador. \\
$\mathbf{2 . 6 1 - 3 . 4 0}$ & Moderada & Indica una moderada presencia de la variable, dimensión, indicador. \\
$\mathbf{1 . 8 1}-\mathbf{2 . 6 0}$ & Baja & Indica una baja presencia de la variable, dimensión, indicador. \\
$\mathbf{1 . 0 0 - 1 . 8 0}$ & Muy baja & Indica una muy baja presencia de la variable, dimensión, indicador. \\
\hline
\end{tabular}

Fuente: Elaboración propia (2017)

\section{RESULTADOS Y DISCUSION}

La tabla 2 presenta los resultados de la dimensión tipos de riesgos y sus indicadores. En general, el resultado de la media aritmética (2.87) indica una moderada presencia de estos tipos de riesgos en las instituciones objeto de estudio. Se observan oportunidades de mejora en acciones asociadas con riesgos físicos, químicos, psicosociales y ambientales los cuales resultaron de moderada presencia y los riesgos biológicos que obtuvieron como resultado baja presencia. 
Tabla 2. Dimensión tipos de riesgos

\begin{tabular}{clcc}
\hline Ítems & & \multicolumn{1}{c}{ Indicadores } & Categoría \\
\hline $\mathbf{1}$ & Riesgos físicos & 2.91 & Moderada presencia \\
$\mathbf{2}$ & Riesgos químicos & 3.20 & Moderada presencia \\
$\mathbf{3}$ & Riesgos biológicos & 2.47 & Baja presencia \\
$\mathbf{4}$ & Riesgos disergonómicos & 2.78 & Moderada presencia \\
$\mathbf{5}$ & Riesgos psicosociales & 3.09 & Moderada presencia \\
$\mathbf{6}$ & Riesgos ambientales & 2.80 & Moderada presencia \\
& Dimensión tipos de riesgos & $\mathbf{2 . 8 7}$ & Moderada presencia \\
\hline
\end{tabular}

Fuente: Elaboración propia (2017)

Al analizar los resultados sobre tipos de riesgos se observa que contrastan con lo formulado por Díaz (2009), para quien los riesgos son factores que inciden en el desenvolvimiento de la organización, los cuales son de naturaleza física, química, orgánica, psicológica o social, La presencia o ausencia de estos pueden relacionarse con la aparición de eventos traumáticos, con efectos crónicos, por lo que las organizaciones deben prevenirlos e implementar planes de acción para evitar o mitigar los mismos.

A continuación, se muestra en la tabla 3 el resumen de la dimensión elementos de la gestión de riesgos.

Tabla 3. Dimensión elementos de la gestión de riesgos

\begin{tabular}{clcc}
\hline Ítems & \multicolumn{1}{c}{ Indicadores } & $\dot{\mathbf{x}}$ & Categoría \\
\hline $\mathbf{1}$ & Políticas de prevención & 3.00 & Moderada presencia \\
$\mathbf{2}$ & Planificación & 2.87 & Moderada presencia \\
$\mathbf{3}$ & Implementación & 2.76 & Moderada presencia \\
$\mathbf{4}$ & Verificación & 2.62 & Moderada presencia \\
$\mathbf{5}$ & Revisión por la dirección & 2.56 & Baja presencia \\
& Dimensión tipos de riesgos & $\mathbf{2 . 7 6}$ & Moderada presencia \\
\hline
\end{tabular}

Fuente: Elaboración propia (2017)

La tabla 3 presenta las medias aritméticas de cada una de las respuestas de los indicadores que conformaban la dimensión elementos de la gestión de riesgos. En general, el resultado de la media aritmética de la dimensión (2.76) compararse con el baremo de interpretación indica una moderada presencia, en las unidades de estudio, de elementos de la gestión de riesgos. De igual manera se observan oportunidades de mejora en los indicadores; políticas de prevención, planificación, implementación, y verificación cuyos resultados fueron de moderada presencia y para el indicador revisión por la dirección que obtuvo resultado de baja presencia. 
En el análisis de los resultados de la dimensión elementos de la gestión de riesgos se observa contraste con lo formulado por Rubio y Rubio. (2006), para quien los elementos de la gestión de riesgos son todas aquellas variables y actividades estratégicas que deben ser aplicadas en la organización, éstas deben estar incluidas como parte de la gestión de la empresa, sin embargo, los resultados muestran que hay moderada presencia de estos elementos en las instituciones estudiadas, además de fallas en el diseño e implementación de los mismos, lo cual llama a las instituciones en estudio a considerar el rediseño e implementación de estos elementos y actividades en concordancia con la legislación aplicable en función de su actividad, de su tamaño y de los riesgos a los que esté sometida, así como de las exigencias que en particular demande la norma, tal cual lo expresa el autor.

En cuanto a la dimensión seguimiento y control de riesgos, se presenta a continuación la tabla 4 donde se muestran los resultados obtenidos.

Tabla 4. Dimensión seguimiento y control de riesgos

\begin{tabular}{clll}
\hline Ítems & \multicolumn{1}{c}{ Indicadores } & $\dot{\mathbf{x}}$ & Categoría \\
\hline $\mathbf{1}$ & Medición y seguimiento del desempeño & 2.47 & Baja presencia \\
$\mathbf{2}$ & Control de registros & 2.22 & Baja presencia \\
$\mathbf{3}$ & Auditorías internas & 2.18 & Baja presencia \\
& Dimensión seguimiento y control de riesgos & $\mathbf{2 . 2 9}$ & Baja presencia \\
\hline
\end{tabular}

Fuente: Elaboración propia (2017)

La tabla 4 presenta el resumen de las medias aritméticas de cada una de las respuestas de los indicadores que conforman la dimensión seguimiento y control de riesgos, la cual obtuvo un resultado de (2.29) que al ser comparado con el baremo de interpretación indica una baja presencia de seguimiento y control de riesgos. Se observan oportunidades de mejora en los indicadores medición y seguimiento del desempeño, control de registros, y auditorías internas los cuales obtuvieron resultados de baja presencia.

En el análisis de los resultados de la dimensión seguimiento y control de riesgos se observa que hay un bajo seguimiento y control de los planes así como de los objetivos planteados por la organización en lo referente a la gestión de riesgos, además se evidencia la no correcta aplicación de estos elementos, lo que limita las acciones para iniciar los cambios necesarios.

Estos resultados son opuestos a lo expresado por el PMBOK (2008), donde se indica que el seguimiento y control son procesos necesarios para supervisar, analizar y regular tanto el progreso como el desempeño, en tanto que mediante la observación y medición sistemática ayudan a identificar las áreas que requieren cambios, así como a dar inicio a los mismos.

En cuanto a la variable en estudio, gestión de riesgos, se presenta a continuación la tabla 5 donde se muestran los resultados obtenidos por las diferentes dimensiones que la conforman. 
Tabla 5. Variable gestión de riesgos

\begin{tabular}{clcc}
\hline Ítems & \multicolumn{1}{c}{ Dimensiones } & $\dot{\mathbf{x}}$ & Categoría \\
\hline $\mathbf{1}$ & Tipos de riesgos & 2.87 & Moderada presencia \\
$\mathbf{2}$ & Elementos de la gestión de riesgos & 2.76 & Moderada presencia \\
$\mathbf{3}$ & Seguimiento y control de riesgos & 2.29 & Baja presencia \\
& Variable gestión de riesgos & $\mathbf{2 . 6 4}$ & Moderada presencia \\
\hline
\end{tabular}

Fuente: Elaboración propia (2017)

La tabla 5 presenta el resumen de medias aritméticas de cada una de las dimensiones que conforman la variable gestión de riesgos, cuyo resultado (2.64) al ser comparado con el baremo e interpretación arrojó como resultado una moderada presencia de gestión de riesgos. Se observan oportunidades de mejora en las dimensiones: Tipos de riesgos y elementos de la gestión de riesgos para los cuales sus resultados (2.87 y 2.76 respectivamente), indican moderada presencia; así como también para la dimensión seguimiento y control de riesgos cuyo resultado (2.29) la ubicó en la categoría de baja presencia.

Al analizar los resultados obtenidos en las tres (3) dimensiones que conforman la variable gestión de riesgos, se puede comprobar que éstos contrastan con lo expresado en la norma COVENIN (3661:2001), donde se plantea la gestión de riesgos como la planificación, desarrollo e implementación de una propuesta para prevenir o evitar, mitigar o reducir el riesgo en una organización, instalación, localidad o en una región. Sin embargo, en las instituciones estudiadas, no se evidencian propuestas referentes a la seguridad, y existe bajo seguimiento y control de la gestión de riesgos por parte de la organización, lo cual conlleva a que, los planes de gestión de riesgos no estén debidamente enfocados en las necesidades que el personal presenta; ni orientados a las medidas de prevención, a que se apliquen los elementos de gestión necesarios para atacar los riesgos ni tampoco a que se hagan las correcciones necesarias a tiempo.
Luego del análisis de los resultados, se hace necesario destacar que los mismos dejan ver la problemática formulada al inicio de la investigación, cuando se planteó que las instituciones públicas de educación universitaria del municipio Cabimas no cuentan con la adecuada gestión de riesgos en el ambiente laboral, lo que causa un déficit en la cultura de prevención de riesgos y en general condiciones de seguridad poco apropiadas en las diferentes áreas de estudio, trabajo, y esparcimiento.

\section{CONCLUSIONES}

Los resultados de la dimensión tipos de riesgos indican moderada presencia de los mismos en las instituciones objeto de estudio, con oportunidades de mejora en acciones asociadas con riesgos físicos, químicos, psicosociales y ambientales los cuales resultaron de moderada presencia así como también para los riesgos biológicos que obtuvieron como resultado baja presencia.

En cuanto a la dimensión elementos de la gestión de riesgos el resultado alcanzado muestra una moderada presencia de los elementos de la gestión de riesgos, con oportunidades de mejora en los indicadores: Políticas de prevención, planificación, implementación, y verificación cuyos resultados fueron de moderada presencia al igual que para el indicador revisión por la dirección que obtuvo resultado de baja presencia.

En relación con la dimensión seguimiento y control de riesgos su resultado de baja presencia evidencia que hay un bajo seguimiento y control 
de los planes así como de los objetivos planteados por la organización en lo referente a la gestión de riesgos, además se pone de manifiesto la poca o no correcta aplicación de estos elementos, lo cual limita las acciones para iniciar los cambios necesarios de seguimiento y control de riesgos en las instituciones públicas de educación universitaria del municipio Cabimas. Sin embargo existen oportunidades de mejora para los indicadores medición y seguimiento del desempeño, control de registros, y auditorías internas los cuales obtuvieron resultados de baja presencia.

Finalmente, el resultado de la variable en estudio, gestión de riesgos, la ubica en la categoría de moderada presencia de gestión de riesgos, mientras que al mismo tiempo presenta posibilidades de mejora para las dimensiones: tipos de riesgos, elementos de la gestión de riesgos y seguimiento y control de riesgos.

Este resultado pone de manifiesto que en las instituciones estudiadas no se evidencian propuestas referentes a la seguridad y hay bajo seguimiento y control de la gestión de riesgos por parte de la organización, lo cual conlleva a que, los planes de gestión de riesgos no estén debidamente enfocados en las necesidades del personal, no están orientados a las medidas de prevención ni a que se apliquen los elementos de gestión necesarios para atacar los riesgos así como tampoco a que se hagan oportunamente las correcciones necesaria.

\section{REFERENCIAS}

Atehortua, F., Bustamante, R. y Valencia, J. (2008). Sistema de gestión integral. Una sola gestión, un solo equipo. Universidad de Antioquia editores. Colombia

Barbat A. (2005), Sistemas de indicadores para la evaluación de riesgos. Universidad Nacional de Colombia. Bogotá. Colombia

Comisión Venezolana de Normas Industriales COVENIN (2270:2002). Comités de higiene y seguridad en el trabajo. Guía para su integración y funcionamiento Requisitos. Fondonorma. Venezuela
Comisión Venezolana de Normas Industriales COVENIN (3661:2001). Gestión de riesgos emergencias y desastres. Definición de términos. Fondonorma. Venezuela.

Cortés, J. (2007). Seguridad e higiene del trabajo. Técnicas de prevención de riesgos laborales. Editorial Tebar. Madrid, España

Delgado, J. (2007). Gestión Integral para la Reducción de los Riesgos Ambientales. CENAMB-UCV. Caracas. Venezuela

Díaz, P. (2009). Prevención de riesgos laborales. Seguridad y salud laboral. Primera edición. Editorial Paraninfo S.A. España

González, N. (2008) Seguridad e Higiene Industrial: Principios Gerenciales. Segunda edición. Edición Venezolana. Maracaibo. Venezuela

Guía del PMBOK. (2008). Guía de los Fundamentos para la Dirección de Proyectos. Project Management Institute, Inc. Pennsylvania. EE.UU

Heerkens, G. (2007). Gestión de proyectos. Primera Edición. Editorial McGraw-Hill. México

Heldman, K. (2005). Risk management. Primera edición. Editorial Jhon Wyley \& Sons. EEUU

Hernández, A; Malfavon, N; y Fernández, G. (2012). Seguridad e higiene industrial. Editorial Limusa. México, México

Kerzner, H. (2009). Project Management; a systems approach to planning, scheduling, and controlling. Décima edición. Editorial Jhon Wyley \& Sons. Nueva Jersey. EEUU.

Ley de Educación Universitaria. Gaceta Oficial $\mathrm{N}^{\circ} 1.429$ (Extraordinaria) de fecha 8 de septiembre de 1970. Caracas. Venezuela

Ley Orgánica de Prevención, Condiciones y Medio Ambiente de Trabajo (2005). Gaceta Oficial $\mathrm{N}^{\circ} 38.236$ del 26 de julio de 2005. Caracas. Venezuela

López, I. (2013). Prevención de riesgos laborales. Primera edición. Editorial Universidad Internacional de la Rioja S.A. España

Norma ISO (9004:2009). Gestión para el éxito sostenido de una organización. Organización Internacional de Normalización. Ginebra. Suiza.

Norma ISO (31000:2009). Gestión del riesgo. Principios y directrices. Organización Internacional de Normalización. Ginebra. Suiza 
Norma OHSAS (18001:2007). Sistema de gestión de seguridad y salud ocupacional. Requisitos. Caracas. Venezuela

Organización Internacional del Trabajo (2010). Lista de enfermedades profesionales. Disponible en: http//es.slideshare.net/nathaliauzcategui/lis ta-de-enfermedadesprofesionales-oit-revision2010. Consultado en diciembre de 2016.

Pérez Porto, J. y Merino, M. (2016). Definición de riesgo ambiental. Disponible en: http://definicion.de/riesgo-ambiental/. Consultado en diciembre de 2016

Pinar Quezada, A. (2009). Conceptos introductorios de Gestión de Riesgos. Universidad Técnica Federico Santa María. Valparaíso. Chile
Programa de las Naciones Unidas para el Desarrollo (PNUD 2012). Conceptos Generales sobre Gestión del Riesgo de Desastres y Contexto del País. Chile.

Rubio, C. y Rubio, M. (2006). Manual de coordinación de seguridad y salud en las obras de construcción. Ediciones Díaz de Santos. Madrid. España

Sistema Normativo de Información Laboral. (2008). Norma Básica de Ergonomía y de Procedimiento de Evaluación de Riesgo Disergonómicos. Universidad de Lima. Perú

Soria, J; Viñas J; Díaz M. (2010). Manual Para la Formación en Prevención de Riesgos Laborales. Editorial Lex Nova. Valladolid. España 